\title{
Competencias de liderazgo estratégico del poder espacial de la Fuerza Aérea Colombiana en escenarios inciertos del futuro ${ }^{1}$
}



https://doi.org/10.21830/9789585380226.02

\author{
Juan Camilo Núnez Cuevas \\ Escuela de Postgrados de la Fuerza Aérea Colombiana
}

Resumen. En esta investigación se identifican las competencias de liderazgo estratégico relevantes para que los integrantes de la Fuerza Aérea Colombiana (FAC) enfrenten los desafíos propios de las futuras amenazas a la seguridad y la defensa desde una perspectiva espacial. Con este propósito se diseñó una novedosa metodología que contempló variables cualitativas y cuantitativas de las ciencias militares, la seguridad y la defensa, competencias, gerencia en macroentornos y de procesos de creación de escenarios futuros. Estas variables fueron procesadas con herramientas metodológicas, técnicas para el fortalecimiento de la objetividad y, finalmente, con la validación de 24 expertos, quienes fueron divididos en dos grupos de experticia: espacio exterior y competencias. Como resultado de la investigación se proponen cuarenta competencias de liderazgo estratégico en escenarios inciertos del futuro para que se incorporen a los procesos formativos de las actuales generaciones que integrarán el poder espacial de la FAC. Asimismo, se concluye que la metodología empleada puede ser usada por cualquier organización e institución que requiera desarrollar en su talento humano las competencias necesarias para enfrentar la incertidumbre del futuro.

Palabras claves: competencias; defensa; educación; espacio exterior; estrategia; Fuerzas Armadas de Colombia; futuro; liderazgo.

1 Este capítulo forma parte de los resultados del proyecto de investigación "Competencias de liderazgo estratégico del poder espacial de la FAC en escenarios inciertos del futuro", de la Escuela de Postgrados de la Fuerza Aérea Colombiana (Epfac). Los puntos de vista y los resultados presentados en este capítulo pertenecen al autor y no reflejan necesariamente los de las instituciones participantes.

2 Mayor de la Fuerza Aérea Colombiana. Magíster en Ciencias Militares Aeronáuticas de la Epfac. Especialista en Gerencia de la Seguridad Operacional del Instituto Militar Aeronáutico (ImA-FAC). OrCID: https://orcid.org/0000-0003-1631-2327_- Contacto: juan.nunez@fac.mil.co 


\section{Introducción}

El futuro ha sido estudiado desde diversos ángulos que han establecido posturas académicas, científicas y sociales, para responder a las dos principales razones por las que el ser humano se ha interesado en él. Al respecto, Höjer y Mattsson (2000) aseguran que la primera razón es que el ser humano desea saber lo que le traerá el futuro y, segundo, porque considera que al conocerlo, lo puede planear, y planeando se genera desarrollo. Este interés le ha permitido al ser humano crear teorías y técnicas para generar tendencias, cálculos, pronósticos y prospectivas, las cuales le dan la capacidad de prepararse. Precisamente, la preparación ante el futuro es el núcleo de esta investigación.

Teniendo en cuenta que la seguridad y la defensa de una nación deben ser preparadas para el futuro, este trabajo pretende responder la siguiente pregunta de investigación: ¿Qué competencias de liderazgo estratégico se deben promover para formar los oficiales que liderarán el poder espacial de la Fuerza Aérea Colombiana (FAC) en escenarios inciertos del futuro? Para ello, la investigación profundiza en un marco teórico que contempla el pensamiento estratégico, la estrategia, el liderazgo, el liderazgo estratégico, las competencias y el poder espacial, soportado en conceptos y estudios sobre el futuro, el liderazgo y en la seguridad y la defensa.

De acuerdo con Ang et al. (2019), la seguridad y la defensa deben visionar y recalibrar en todo momento las necesidades de competencias para el liderazgo, que según Iso-Markku y Müller-Brandeck-Bocquet (2019) debe adaptarse como una cultura estratégica a las nuevas necesidades que se puedan vislumbrar. De esta manera, al concebir la idea de una seguridad multidimensional con nuevas perspectivas (Álvarez, 2017), las competencias de liderazgo pueden enfrentar la incertidumbre de las amenazas y la naturaleza de las crisis modernas, para que los líderes puedan adquirir nuevas habilidades y, como afirman Chua et al. (2019), pensar en solucionar futuras crisis, ya que en relación con el futuro no hay marcos que limiten el pensamiento (Air Force Space Command, 2019).

El estudio de estas temáticas permite comprender que no existe un método estándar y rígido para crear competencias, lo cual motiva a idear una 
metodología de investigación propia para un estudio mixto, con uso de herramientas metodológicas de macroentornos, proyección de escenarios y validación por 24 expertos, para proponer como resultado final unas competencias de liderazgo estratégico en escenarios futuros.

El proceso investigativo sobre el problema permite generar una hipótesis que se sustenta en dos suposiciones principales: primero, que se requieren muchos años para desarrollar competencias en el ser humano, y segundo, que las instituciones de seguridad y defensa de las naciones deben proyectar y vislumbrar escenarios inciertos del futuro y propender a formar con suficiente tiempo de anticipación las competencias de sus futuros líderes. De esta manera, logran que estos puedan enfrentar la incertidumbre del futuro y garantizar el éxito de la seguridad y la defensa en escenarios volátiles, inciertos, complejos y ambiguos.

\section{Métodos}

Este estudio se desarrolló mediante la identificación de influencias externas de macroentornos que retaron al liderazgo estratégico espacial años atrás, las cuales se combinaron con herramientas de creación de escenarios inciertos y un Modelo Básico Común de creación de competencias, para proponer competencias de liderazgo estratégico del futuro. Estas competencias fueron filtradas por un grupo de expertos en comportamiento humano y un segundo grupo compuesto por expertos en espacio exterior, lo cual permitió identificar las competencias de liderazgo estratégico que potencialmente en un futuro cualquier organización espacial requerirá de sus líderes.

\section{Paradigma, tipo de estudio y marco teórico}

Siguiendo la recomendación de Fernández et al. (2006), esta investigación determinó un paradigma de enfoque integrado multimodal para responder a preguntas sobre el futuro. Además, siguiendo el trabajo de Robledo et al. (2004), se aplicó un tipo de estudio mixto que permitiera procesar diversos tipos de información cualitativa y cuantitativa para cumplir con las exigencias del objetivo general. 
El marco teórico investigado contempló teorías del liderazgo, como del gran hombre, la teoría del rasgo, la teoría del comportamiento, la teoría de la contingencia, la teoría del liderazgo situacional, la teoría del líder seguidor, la teoría del liderazgo transformacional y la teoría del liderazgo del sistema. En el ámbito de las competencias, se estudiaron las teorías del efecto, las ejecutivas, las del comportamiento, las técnicas HвP (Human Behavior Performance), las del liderazgo estratégico del saber, del hacer, de las tres habilidades básicas y las del cluster outstanding. En relación con la seguridad y la defensa, las teorías aportadas por Raybourn et al. (2017) permitieron identificar la importancia de usar la innovación y el aprendizaje para propiciar el crecimiento y el desarrollo en el liderazgo en todos los escalones.

Además de lo anterior, los métodos de captura de información se enriquecieron con los aportes de Aguayo et al. (2018), quienes afirmaron que el pilar para liderar en situaciones extremas es el nivel de entrenamiento en estas capacidades. De la misma manera, fue tenida en cuenta la teoría del liderazgo estratégico propuesta por Shao (2019), como reflejo de bases cognitivas y valores de los tomadores de decisiones. Por último, también se tuvieron en cuenta las reglas para sostener el liderazgo en la revolución digital estudiadas por Leavy (2020).

En cuanto a las competencias de organizaciones aeroespaciales, se contemplaron las teorías de coherencia actitudinal, las del trato digno a las personas, las de proactividad, las del mejoramiento continuo, las de trabajo en equipo, las de formulación estratégica, las de sinergia institucional y las de vocación de servicio. También se estudiaron investigaciones en la relación entre la industria espacial y el desarrollo nacional de la China National Space Administration (2016); la transversalidad de los productos y servicios derivados del poder espacial, que, de acuerdo con Quiroga et al. (2019), ayudan a los ministerios de Defensa a enfrentar los retos y los desafíos; los aportes de Hyten (2016) sobre los entornos operacionalmente limitados, congestionados y degradados; las políticas sobre el espacio exterior expuestas en Francia por The French Ministry for the Armed Forces (2019) para contribuir a la seguridad y el desarrollo de las economías de la sociedad. 
También se tuvieron en cuenta diversos conceptos de la Escuela Superior de Guerra de Colombia, particularmente las estrategias espaciales formuladas por Álvarez y Corredor (2019); los desafíos para la seguridad estudiados por Becerra et al. (2019); las estrategias de defensa espacial de Europa estudiadas por Polkowska (2020) y, finalmente, los dilemas de los escenarios cambiantes que Fernández-Osorio et al. (2020) propusieron recientemente.

\section{Fases metodológicas del proyecto de investigación}

La metodología diseñada (figura 1) permitió responder la pregunta de investigación: ¿Qué competencias de liderazgo estratégico se deben promover para formar los oficiales que liderarán el poder espacial de la FAC en escenarios inciertos del futuro? El diseño de la metodología contempló fases con etapas cualitativas y cuantitativas, y tres herramientas metodológicas: Pestel $+\mathrm{D}$, Scenarios Building y validación de expertos.

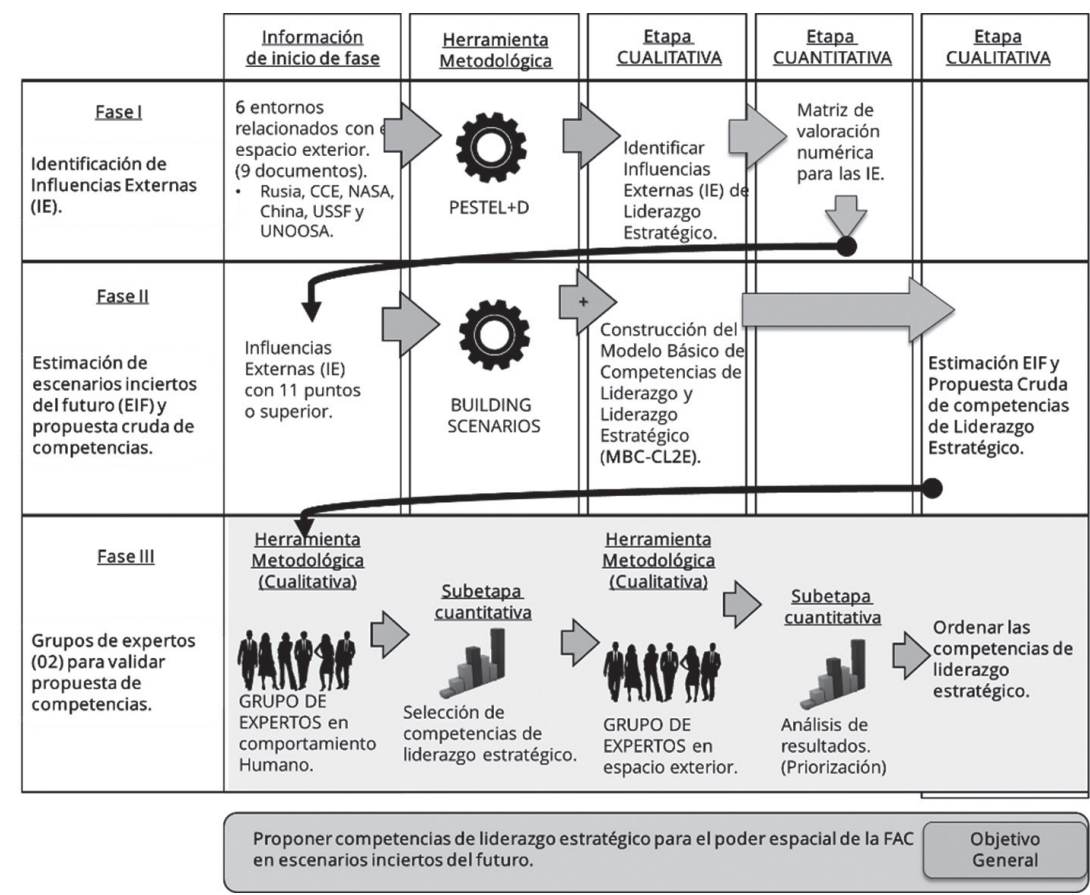

Figura 1 Método de investigación y fases metodológicas del proyecto de investigación. Fuente: Elaboración propia. 


\section{Fase I. Identificación de influencias externas}

Se seleccionaron nueve documentos de los seis entornos relacionados con el espacio exterior (tabla 1), con el propósito de identificar las influencias externas que en determinado momento retaron a sus líderes. En esta investigación se entendió la influencia externa como los retos, problemas, presiones y dificultades que, en debido momento, los líderes estratégicos tuvieron que enfrentar y que cualquier líder de una organización espacial debería saber enfrentar.

Tabla 1. Fuentes de información de los seis entornos relacionados con el espacio exterior y sus nueve documentos

\begin{tabular}{|c|c|c|c|c|}
\hline N. ${ }^{\circ}$ & $\begin{array}{l}\text { Entorno con } \\
\text { espacio }\end{array}$ & Documento & $\begin{array}{c}\text { Fuente }(9 \\
\text { documentos) }\end{array}$ & $\begin{array}{l}\text { Cantidad } \\
\text { de páginas }\end{array}$ \\
\hline \multirow[t]{2}{*}{1} & Rusia & $\begin{array}{l}\text { Law of the Russian Federation } \\
\text { N. } 5663-1 \text { of August } 20,1993 \\
\text { on Space Activities (Addenda } \\
\text { of } 29 / 11 / 1996,10 / 01 / 2003 \text {, } \\
\text { /05/03/2004, 22/08/2004, } \\
02 / 02 / 2006 \text { and 18/12/2006). }\end{array}$ & Federación Rusa & 9 \\
\hline & & $\begin{array}{l}\text { Outer Space in Russia's } \\
\text { Security Strategy. }\end{array}$ & $\begin{array}{l}\text { Simons Papers } \\
\text { in Security and } \\
\text { Development N. } \\
64 / 2018 \mid \text { August } \\
2018\end{array}$ & 22 \\
\hline 2 & Colombia, CCE & $\begin{array}{l}\text { Actas plenarias XIV 20/05/13, } \\
\text { XV 27/11/13 y XVI } \\
\text { 22/04/2014. }\end{array}$ & $\begin{array}{l}\text { Comisión } \\
\text { Colombiana del } \\
\text { Espacio }\end{array}$ & 55 \\
\hline 3 & China, CNSA & China’s White Paper. & China, CNSA & 10 \\
\hline 4 & $\begin{array}{l}\text { Estados Unidos, } \\
\text { NASA }\end{array}$ & Nasa Strategic Plan 2018. & $\begin{array}{l}\text { National Aeronau- } \\
\text { tics and Space } \\
\text { Administration, } \\
\text { Washington, D. C, } \\
20546 .\end{array}$ & 64 \\
\hline 5 & OnU, UnOOSA & $\begin{array}{l}\text { Evaluation of the United } \\
\text { Nations Office for Outer Space } \\
\text { Affairs } 2019 \text {. }\end{array}$ & $\begin{array}{l}\text { United Nations, } \\
\text { Unoosa Assignment } \\
\text { N. } .^{\circ} \text { IED-19-003 }\end{array}$ & 39 \\
\hline 6 & $\begin{array}{l}\text { Estados Unidos, } \\
\text { Department of } \\
\text { Defense, UssF }\end{array}$ & $\begin{array}{l}\text { Space Policy Directive-4, } \\
\text { Establishment of the US Space } \\
\text { Force, 19/02/2019. }\end{array}$ & $\begin{array}{l}\text { Office of the Press } \\
\text { Secretary, Donald } \\
\text { Trump }\end{array}$ & 7 \\
\hline
\end{tabular}

Fuente: Elaboración propia. 
Para extraer las influencias externas, la metodología usó la herramienta metodológica de macroentornos Pestel, defendida recientemente por Pupo et al. (2020), a la cual se le adicionó el factor defensa (Pestel + D), para poder identificar los factores político (P), económico $(\mathrm{E})$, social $(\mathrm{S})$, tecnológico $(\mathrm{T})$, ambiental (E), legal (L) y defensa (D). Para distinguir las influencias externas de mayor importancia, se usó una matriz de valoración numérica que dio un peso específico a cada influencia externa.

\section{Fase II. Estimación de escenarios inciertos del futuro y propuesta cruda de competencias}

Después de lo expuesto, las influencias externas con mínimo 11 puntos fueron procesadas en una herramienta de estimación de escenarios futuros llamada Scenarios Building, la cual logró fusionar las influencias externas con la incertidumbre de escenarios futuros para el poder espacial de la FAC.

Posteriormente, se desarrolló un Modelo Básico Común de Competencias de Liderazgo y Liderazgo Estratégico, llamado MBC-CL2E, el cual permitió crear competencias a partir del estudio de trece investigaciones formuladoras de competencias en los sectores de seguridad, defensa, salud, energía atómica, ciberseguridad, desarrollo, emprendimiento, aeroespacial, multinacional y finanzas internacionales. Acto seguido, se fusionó el MBC-CL2E con las influencias externas identificadas en la fase I y los escenarios estimados con la herramienta Scenarios Building de la fase II, lo cual generó una propuesta cruda de competencias de liderazgo estratégico del futuro. Para que esta propuesta cruda (sin filtro) estuviese libre de posibles sesgos por la percepción del investigador, la metodología proyectó la necesidad de validar esta propuesta por expertos en las áreas de las competencias y de las ciencias espaciales, tarea realizada en la fase III.

\section{Fase III. Grupo de expertos para validar la propuesta de competencias}

La herramienta virtual de validación permitió ejecutar dos validaciones con dos grupos de expertos. El primer grupo de expertos se estructuró con catorce oficiales psicólogos, con experiencia en procesos de potencial humano, quienes filtraron y eliminaron aquellas competencias de la propuesta cruda que no tenían características de competencias de liderazgo estratégico. 
Posteriormente, una vez depurada esta nueva propuesta, el segundo grupo de diez expertos en ciencias espaciales validó cada competencia sugerida y las priorizó en orden de mayor a menor importancia, de cara a la creación de un poder espacial en el futuro. Con este último paso se solucionó el problema de investigación.

\section{Resultados}

La investigación identificó 18 influencias externas, 72 escenarios inciertos del futuro y 40 competencias de liderazgo estratégico, las cuales respondieron a la pregunta de investigación (tabla 2 y figura 2).

Tabla 2. Las cuarenta competencias de liderazgo estratégico para el poder espacial de la Fuerza Aérea Colombiana en escenarios inciertos del futuro validadas por los 24 expertos

\begin{tabular}{cl}
\hline Puntaje & \multicolumn{1}{c}{ Competencia de liderazgo estratégico } \\
\hline 9 & Pensamiento crítico \\
9 & Prospectiva diplomática \\
8 & Diplomacia espacial \\
8 & Influencia de gestión \\
8 & Toma de decisiones sistémicas \\
8 & Motivar e inspirar para crear líderes para el espacio \\
8 & Motivar el talento humano \\
8 & Pensamiento analítico de alianzas \\
8 & Visión 360 \\
8 & Visión, prospectiva y proactividad para el espacio \\
7 & Construcción de alianzas estratégicas pro espacio \\
7 & Persuasión política \\
7 & Persuasión, influencia y diplomacia espacial en VICA \\
7 & Empoderamiento legal del espacio \\
7 & Juicio y comprensión de limitaciones \\
7 & Motivación al emprendimiento \\
7 & Prospectiva de alianzas GeG \\
7 & Prospectiva de la incertidumbre \\
7 & Prospectiva del cambio \\
7 & Prospectiva espacial \\
\hline
\end{tabular}




\begin{tabular}{cl}
\hline Puntaje & \multicolumn{1}{c}{ Competencia de liderazgo estratégico } \\
\hline 7 & Prospectiva para empleo del poder espacial \\
6 & Construcción de alianzas estratégicas pro espacio \\
6 & Construcción de alianzas innovadoras \\
6 & Persuasión para el equilibrio diplomático \\
6 & Persuasión, influencia y diplomacia espacial \\
6 & Gestión del cambio \\
6 & Gestión del cambio para la defensa \\
6 & Juicio para ideas productivas \\
6 & Toma de decisiones de convergencia \\
6 & Visión de políticas públicas \\
5 & Persuasión de equilibrio \\
5 & Toma de decisiones en gestión de proyectos \\
5 & Motivar el aprendizaje \\
4 & Diplomacia en solución de conflictos \\
4 & Diplomacia inteligente \\
4 & Empoderar al autocrítico \\
3 & Prospectiva de cooperación \\
1 & Arte estratégico con enfoque legal \\
1 & Arte estratégico cooperativo \\
\hline
\end{tabular}

Fuente: Elaboración propia.

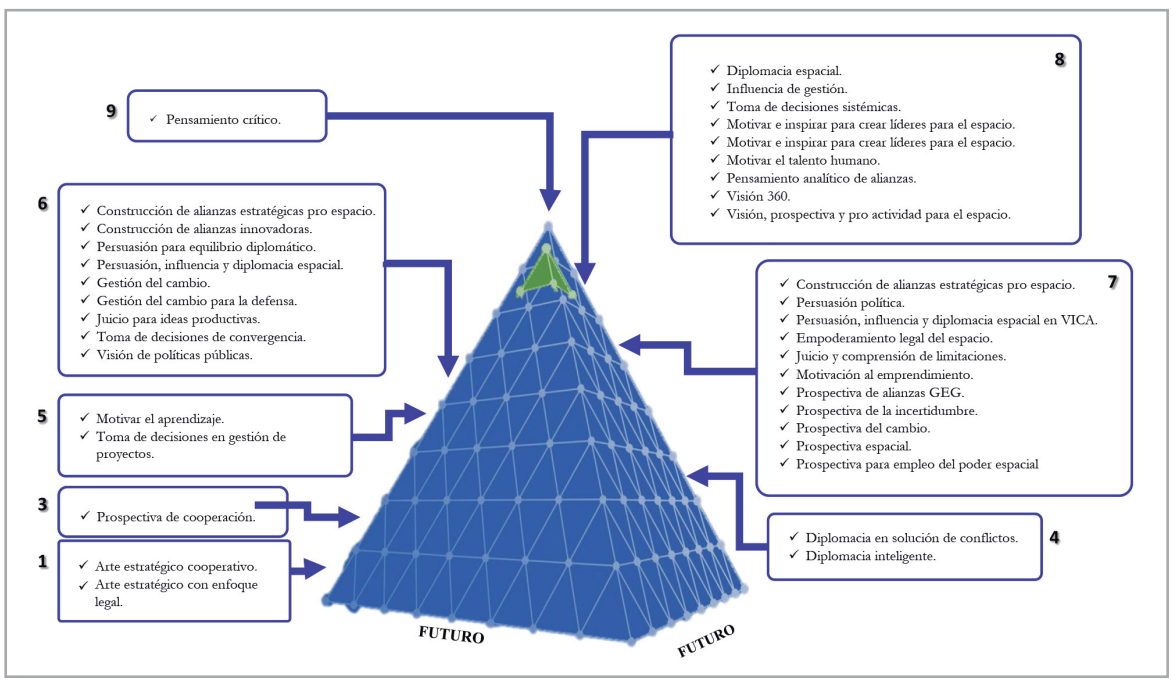

Figura 2. Pirámide de las cuarenta competencias de liderazgo estratégico para el poder espacial de la Fuerza Aérea Colombiana en escenarios inciertos del futuro validadas por los 24 expertos. Fuente: Elaboración propia. 


\section{Discusión}

Como se ha demostrado hasta este momento, la metodología usada, aunque compleja, permitió responder la pregunta de investigación: ¿Qué competencias de liderazgo estratégico se deben promover para formar los oficiales que liderarán el poder espacial de la FAC en escenarios inciertos del futuro?, lo cual se soporta en los resultados demostrados líneas arriba.

Los resultados permiten discutir si es acertado pretender identificar las influencias externas de la experiencia de Rusia, China, la NASA, la Ussf, la CCE y la UnOosa, como fuente de información para vislumbrar escenarios inciertos que los futuros líderes tendrían que enfrentar. Sobre esta parte de la discusión, surgen dos planteamientos. El primero considera que no es una iniciativa descabellada, ya que las influencias externas seleccionadas ofrecieron mayor exigencia a su liderazgo estratégico. El segundo planteamiento, esta vez antagonista, considera que los avances tecnológicos en ciencias espaciales permitirán que el camino que deben recorrer los líderes del futuro no será el mismo de quienes lucharon en la carrera espacial.

Después de la discusión anterior, es necesario resaltar que la investigación optó por el primer planteamiento, porque no se justificaría cometer los mismos errores, y es de inteligentes aprender de la experiencia de los demás. Por otro lado, como dice el título de la investigación, se trata de proponer competencias en escenarios inciertos, es decir, no hay escenarios verídicos y exactos, solo proyectados y estimados. Además, hay que tener en cuenta que en el ámbito de la seguridad y la defensa, la transferencia de experiencia e información siempre es limitada, sesgada o parcial.

Los hallazgos de esta investigación se consideran evidencia científica, afirmación que se justifica en el hecho de que cada una de las cuarenta competencias propuestas posee una relación directa con alguno de los seis entornos relacionados con el espacio exterior. Es interesante elucubrar al respecto y profundizar en esta parte de la discusión, ya que, por ejemplo, dos de las competencias con más puntaje fueron C-CHINA-42 "pensamiento crítico" y C-1-RuSIA-SissF-12 "prospectiva diplomática", las cuales provienen directamente de influencias externas de China — cuando se dio cuenta de que otras 
potencias habían construido sólidas alianzas entre ellas en el ámbito políticoy de Rusia — cuando percibió directamente que los Estados Unidos estaba fortaleciendo su estrategia espacial desde la perspectiva de la seguridad y la defensa-.

Para concluir este aparte, se defiende a ultranza la metodología usada, aunque podría ser más simple o corta, motivo de posibles discusiones futuras, pero es innegable que esta responde al problema de estudio. La metodología puede ser usada por cualquier organización e institución que requiera desarrollar en su talento humano las competencias necesarias para enfrentar la incertidumbre del futuro, ya que no se podrá ocultar o negar que la proyección de competencias es una tarea que siempre se debe desarrollar con suficiente tiempo, responsabilidad y profesionalismo.

\section{Conclusiones}

El desarrollo de competencias de liderazgo estratégico no sucede en corto tiempo, de manera que es acertado que una organización de seguridad y defensa proyecte las competencias que sus futuros líderes puedan necesitar ante escenarios proyectados.

La estimación de escenarios futuros para la seguridad y la defensa se puede basar en experiencias propias y ajenas, siempre y cuando esta estimación se realice con herramientas de prospectiva o de creación de escenarios acordes a las problemáticas de la organización.

Identificar competencias de liderazgo para el futuro facilita la proyección de las organizaciones y su permanencia exitosa en el tiempo. Las ciencias militares tienen una relación directa con las competencias, ya que el militar, como estratega y tomador de decisiones, decide también sobre las competencias que requiere que sus subordinados posean para enfrentar escenarios inciertos del futuro.

Esta investigación propone un modelo generador de competencias que puede ser usado por cualquier organización que considere imperativo preparar a las actuales generaciones ante la incertidumbre del mańana. 


\section{Referencias}

Aguayo, M., Jiménez, S., \& Martillo, I. (2018). El pensamiento crítico y su relación con el pensamiento estratégico y el liderazgo. Revista Universidad y Sociedad, 10(3), 112-116.

Air Force Space Command. (2019). The future of space 2060 and implications for U.S. strategy: Report on the space futures workshop. Office of the Chief Scientist. https://apps. dtic.mil/sti/pdfs/AD1095527.pdf

Álvarez, C. (Ed.). (2017). Escenarios y desafíos de la seguridad multidimensional en Colombia (vol. 1). Escuela Superior de Guerra. https://doi.org/10.25062/9789585652835

Álvarez, C. E., \& Corredor, C. G. (2019). Mirando hacia las estrellas: Una constante necesidad humana. El espacio exterior: una oportunidad infinita para Colombia. Escuela Superior de Guerra.

Ang, J., Chen, W., Tan, C., \& Yee, L. (2019). Defining the leader in border security: A leadership competency framework. Home Team Journal, (8), 145.

Becerra, J., Sánchez, M., Castañeda, C., Bohórquez-Keeney, A., Páez, R., Baldomero, A., \& León, I. (2019). La seguridad en el ciberespacio: Un desafio para Colombia (1. ${ }^{a}$ ed.). Escuela Superior de Guerra; Ministerio de Tecnologías de la Información y las Comunicaciones. https://doi.org/10.25062/9789585216549

China National Space Administration (CNSA). (2016). China's Space Activities in 2016. http://www.china.org.cn/government/whitepaper/node_7245058.htm

Chua, S., Khader, M., \& Tan, E. (2019). Leading in a fat tail world: Modern crises and recommendations for leaders. Home Team Journal, (8), 145.

Fernández, C., Baptista, P., \& Hernández, R. S. (2006). Metodología de la investigación (4. ${ }^{a}$ ed.). McGraw Hill.

Fernández-Osorio, A., Miron, M., \& Ramírez, L. (2020). Dilemas de los escenarios cambiantes en seguridad y defensa. Revista Cientifica General José María Córdova, 18(31), 477-479. https://doi.org/10.21830/19006586.658

Höjer, M., \& Mattsson, L. (2000). Determinism and backcasting in future studies. Futures, 7(32), 613-634. https://doi.org/10.1016/S0016-3287(00)00012-4

Hyten, J. E. (2016). Space mission force: Developing space warfighters for tomorrow. White paper. Air Force Space Command.

Iso-Markku, T., \& Müller-Brandeck-Bocquet, G. (2019). Towards German leadership? Germany's evolving role and the EU's Common Security and Defence Policy. German Politics, 1-20. https://doi.org/10.1080/09644008.2019.1611782

Leavy, B. (2020). Amit mukherjee: seven principles shaping. Strategy \& Leadership, 48(3), 33-38.

Polkowska, M. (2020). Space defense in Europe. Policy and Security Aspects. Polish Political Science Yearbook, 49(2), 127-139. https://doi.org/10.15804/ppsy2020207 PL ISSN 0208-7375

Pupo, A. R., Ponce, D. V., Viteri, G. F., \& Bustillo, F. S. (2020). PESTEL analysis of environment State responsibility in Ecuador. Neutrosophic Sets and Systems, 34(1), 70-78. 
Quiroga, R. S., Gutiérrez, N. P., Núńez, J. C., \& Rico, Y. (2019). El espacio exterior: Una oportunidad infinita para Colombia. La Fuerza Aérea Colombiana y la evolución del pensamiento estratégico (1. ${ }^{\mathrm{a}} \mathrm{ed}$.). Escuela Superior de Guerra.

Raybourn, E. M., Schatz, S., Vogel, J., \& Vierling, K. (2017). At the tipping point: Learning science and technology as key strategic enablers for the future of defense and security. Interservice Industry Training, Simulation, and Education Conference, 17(109), 1-12.

Robledo, L. R., Arcila, A., Buriticá, L. H., \& Castrillón. J. (2004). Paradigmas y modelos de investigación (2. ${ }^{\mathrm{a}}$ ed.). Fundación Universitaria Luis Amigó.

Shao, Z. (2019). Interaction effect of strategic leadership behaviors and organizational culture on IS-business strategic alignment and enterprise systems assimilation. International Journal of Information Management, 44(2), 96-108.

The French Ministry For The Armed Forces. (2019). Space defense strategy. https://www.defense. gouv.fr/content/download/574375/9839912/Space\%20Defence\%20Strategy\%202019_ France.pdf

\section{Bibliografía consultada}

American Association of Colleges of Pharmacy. (2011). Core competencies for interprofessional collaborative practice. Interprofessional Education Collaborative.

Apollo Education Group \& University of Phoenix. (2015). Competency models for enterprise security and cybersecurity. http://www.apollo.edu/content/dam/apolloedu/microsite/ security_industry/AEG-UOPX\%20Security\%20Competency\%20Models\%20report.pdf

Bacigalupo, M., Kampylis, P., Punie, Y., \& Van, G. (2016). EntreComp: The entrepreneurship competence framework ( $1^{\mathrm{a}}$. ed.). Joint Research Centre.

Chartered Global Management Accountant. (2020). CGMA competency framework 2019 edition. https://www.cgma.org/resources/tools/cgma-competency-framework.html

Grachev, M. V., \& Bobina, M. A. (2006). Russian organizational leadership: Lessons from the globe study. International Journal of Leadership Studies, 1(2), 67-79.

International Atomic Energy Agency. (2018). The competency framework. https://www.iaea. $\mathrm{org} /$ sites/default/files/18/03/competency-framework.pdf

International Labour Organization. (2015). Regional model competency standards: Core competencies. https://www.ilo.org/wcmsp5/groups/public/---asia/---ro-bangkok/documents/publication/wcms_420961.pdf

Jackson, N. K. (2018). Outer space in Russia's security strategy. School for International Studies, 64(2), 1- 22.

Malloch, K., \& Mazurek, B. M. (2013). Developing high-level change and innovation agents competencies and challenges for executive leadership. Nurs Admin Q, Wolters Kluwer Health, 37(1), 7 (60-66).

National Aeronautics and Space Administration [NASA]. (2018). NASA strategic plan 2018. https://www.nasa.gov/sites/default/files/atoms/files/nasa_2018_strategic_plan.pdf 
38 Volumen I. Panorama en seguridad y defensa visto desde las instituciones de educación superior de las Fuerzas Armadas.

Office of Internal Oversight Services [OIOS]. (2019). Evaluation of the United Nations Office for Outer Space Affairs. https://oios.un.org/file/7742/download?token=uPFNsUU2

Office of Space Commerce. (2019). Space policy directive-4, establishment of the United States Space Force. https://www.space.commerce.gov/policy/national-space-council-directives/

United Nations Development Programme. (2016). Core competency framework. https:// www.undp.org/content/dam/undp/library/corporate/Careers/undp-hr-core-competency-2016.pdf

United States Office of Personnel Management. (2012). Guide to senior executive service qualifications. https:/www.opm.gov/policy-data-oversight/senior-executive-service/reference-materials/guidetosesquals_2012.pdf

Van, M., \& Grachev, M. (2010). Building strategic leadership competencies: The case of Unilever. International Journal of Leadership Studies, 5(3), 317-332.

Wong, L., Stephen, G., Kidd, W., Pricone, R., \& Swengros, R. (2003). Strategic leadership competencies. Army War College.

Yeltsin, B. (1993). Law of the Russian Federation No. 5663-1 of august 20, 1993 on space activities. https://www.wto.org/english/thewto_e/acc_e/rus_e/WTACCRUS58_LEG_375.pdf 